\title{
Forming nanoparticles of water-soluble ionic molecules and embedding them into polymer and glass substrates
}

\author{
Stella Kiel ${ }^{1}$, Olga Grinberg ${ }^{1}$, Nina Perkas ${ }^{1}$, Jerome Charmet $^{2}$, \\ Herbert Kepner $^{2}$ and Aharon Gedanken ${ }^{*}, \S$
}

\section{Full Research Paper}

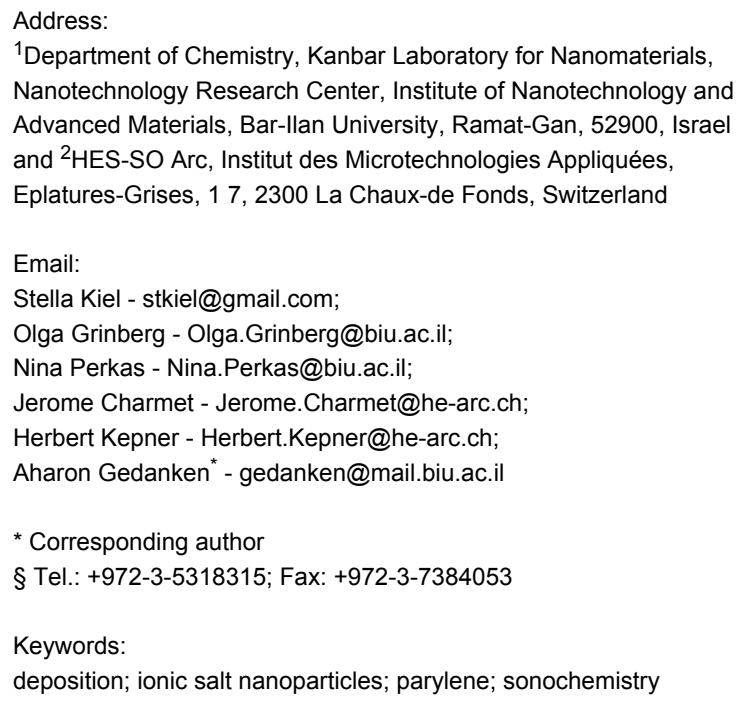

${ }^{1}$ Department of Chemistry, Kanbar Laboratory for Nanomaterials, Nanotechnology Research Center, Institute of Nanotechnology and Advanced Materials, Bar-Ilan University, Ramat-Gan, 52900, Israel and ${ }^{2} \mathrm{HES}-\mathrm{SO}$ Arc, Institut des Microtechnologies Appliquées, Eplatures-Grises, 17, 2300 La Chaux-de Fonds, Switzerland

\begin{abstract}
This work describes a general method for the preparation of salt nanoparticles (NPs) made from an aqueous solution of ionic compounds $\left(\mathrm{NaCl}, \mathrm{CuSO}_{4}\right.$ and $\left.\mathrm{KI}\right)$. These nanoparticles were created by the application of ultrasonic waves to the aqueous solutions of these salts. When the sonication was carried out in the presence of a glass microscope slide, a parylene-coated glass slide, or a silicon wafer the ionic NPs were embedded in these substrates by a one-step, ultrasound-assisted procedure. Optimization of the coating process resulted in homogeneous distributions of nanocrystals, $30 \mathrm{~nm}$ in size, on the surfaces of the substrates. The morphology and structure of each of the coatings were characterized by physical and chemical methods, such as X-ray diffraction (XRD), scanning electron microscopy (SEM), atomic force microscopy (AFM), Raman spectroscopy and X-ray photoelectron spectroscopy (XPS). After $24 \mathrm{~h}$ of leaching into water the nanoparticles of the inorganic salts were still present on the slides, and complete leaching of nanoparticles occurred only after $96 \mathrm{~h}$. A mechanism of the ultrasound-assisted coating is proposed.
\end{abstract}




\section{Introduction}

The incorporation of nanocrystals into dielectric matrices, such as glass or polymers, has become a topic of broad interest in recent years. The research in the area of nanostructured composites is aimed at studying their fundamental properties as well as applications in tissue engineering, nanooptics and nanoelectronics [1-5].

Unlike the synthesis of NPs of metal oxides, metal chalcogenides, and even some metal fluorides, the synthesis of NPs of water-soluble ionic compounds has not reached the same level of attention, and only a very few publications are found [6] This current paper describes a general method for the preparation of water-soluble ionic NPs. To help prove the formation of these NPs they were examined both in the solution in which they were formed as well as in solid matrices. They were embedded in the solid substrate by the sonochemical method, which was performed subsequently to their formation.

Various methods have been used for the incorporation and growth of arrays of nanocrystals on or embedded into substrate hosts. One of these methods is chemical bonding. For example, the cross-linking process was used for the embedding of $\mathrm{NaCl}$ nanocrystals into polymers containing unsaturated double bonds $[1,2]$.

Some other methods were developed for the coating of polymers with nanocrystals of $\mathrm{NaCl}, \mathrm{Na}_{2} \mathrm{CO}_{3}$ and $\mathrm{Na}_{2} \mathrm{SO}_{4}$, exploiting the difference in wettability between the regions of the patterned polymeric substrate [3]. Suh et al. [3] fabricated single nanocrystal arrays of various sizes on sub-microwells of poly (ethylene glycol) copolymer, using selective wetting of the hydrophilic regions of the exposed substrate surface and subsequent drying. The single $\mathrm{NaCl}$ nanocrystals in the range of 100-150 nm were prepared by immersion of a concavepatterned polystyrene film in aqueous $\mathrm{NaCl}$ solution, which was then lifted out of the solution slowly [4]. A new synthesis route was developed by Malfatti et al. for the formation of $\mathrm{NaCl}$ nanoboxes on a silicon wafer and silica glass slide as substrate, through an evaporation-induced self-assembly process [7].

The insertion of nanocrystals into polymer and glass substrates was also performed by some physical methods. The incorporation of $\mathrm{NaCl}$ nanocrystals into porous glass by high-pressure injection was reported by Parfen'eva et al. [5], and a change of the thermal conductivity of the substrate was demonstrated. The embedding of $\mathrm{NaCl}$ nanoparticles into a polymeric film was recently realized by the use of an electromagnetic field [8] According to this method, the nanoparticles absorbed energy upon illumination by laser light, converted it to heat, and sank into a locally softened polymer film, as probed by atomic force microscopy. Lomonosov et al. [9] used an ultrasonic piezo dispenser for the introduction of $\mathrm{NaCl}$ nanoparticles from $1 \mathrm{M}$ aqueous $\mathrm{NaCl}$ onto mica slides and demonstrated regions with a regular $\mathrm{NaCl}$ nanocrystal structure, by AFM. These structures varied from rows of single square nanocrystals to rows of long rectangular crystals with average sizes of $300 \mathrm{~nm}$ in width, $60 \mathrm{~nm}$ in height and up to several micrometers long. The structural changes were explained as being due to surface diffusion; however, the mechanism of the deposition, the attachment of $\mathrm{NaCl}$ nanoparticles and the formation of the crystalline structure were not explicitly discussed [9].

We have reported on the sonochemical deposition of inorganic nanoparticles on and into various materials and demonstrated that the ultrasound assisted method is effective in uniform distribution and strong attachment of nanoparticles to the surfaces of the substrates and even their penetration into the solid body [10]. These observations can be explained as a result of the extreme conditions of high pressure (>1000 atm) and temperature $(>500 \mathrm{~K})$ developed during the collapse of the acoustic bubble. The after effect of this collapse near a solid surface is the formation of high-speed microjets and shock waves in the liquid, directed towards the solid surface. These microjets throw the just-formed nanoparticles toward the substrate at such a high speed $(>500 \mathrm{~m} / \mathrm{s})$ that it leads to the strong adherence of the NPs to the surface without the need for any binding agents $[11,12]$.

In the present work we report for the first time on a novel general method for the preparation of water-soluble NPs of ionic salts. The method was applied to $\mathrm{NaCl}, \mathrm{CuSO}_{4}$, and $\mathrm{KI}$. The general method suggested for the preparation of watersoluble ionic NPs is sonochemistry. The salt nanocrystals of $\mathrm{NaCl}, \mathrm{CuSO}_{4}$ and $\mathrm{KI}$ were immobilized from the aqueous solution of the dissolved ionic compounds onto the microscope glass slides, parylene-coated glass slides, and silicon wafers. This was accomplished by a one-step, ultrasound-assisted procedure. The ionic nanoparticles were thrown to the solid surface by the sonochemical microjets and were strongly anchored to the substrates. The coated substrates were characterized by chemical and physical methods.

\section{Experimental \\ Materials}

All of the chemical reagents were purchased from Sigma Aldrich and were of analytical chemical purity and used without further purification. The glass microscope slides were purchased from Laborderate GmbH Germany. The parylene-Ccoated glass microscope slides were received from COMELEC SA (Switzerland), and the procedure for their preparation has 
been described previously [13]. The thickness of the parylene layer was in the range of 6-12 $\mu \mathrm{m}$. Each glass slide was cut to a size of 1 inch $^{2}$ to be inserted into the reaction vessel.

\section{Deposition of $\mathrm{NaCl} / \mathrm{CuSO}_{4} / \mathrm{KI}$ on regular/parylene- coated glasses and on silicon wafer/parylene- coated silicon wafer}

Typically, $\mathrm{NaCl}$ was dissolved in doubly distilled water to obtain a concentration in the range of 0.04-0.5 M. The dissolution of the $\mathrm{NaCl}, \mathrm{CuSO}_{4}$ and $\mathrm{KI}$ was achieved by magnetic stirring of the solutions for $30 \mathrm{~min}$ at room temperature. The sonochemical treatment was carried out in the presence of glass/ parylene-coated glass by immersion of the high-intensity $\mathrm{Ti}$ horn $(20 \mathrm{kHz}, 750 \mathrm{~W})$ into the reaction vessel (Figure 1). The reaction time varied from $15 \mathrm{~min}$ to $90 \mathrm{~min}$. The efficiency of the ultrasonication was in the range of $23-30 \%$. The deposition of $\mathrm{CuSO}_{4}$ and $\mathrm{KI}$ was achieved by the same procedure. All the experiments were repeated three times and good reproducibility of the results was demonstrated.

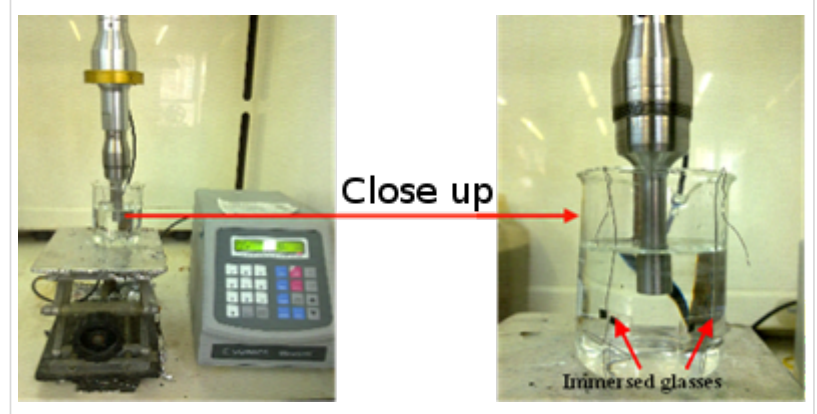

Figure 1: Coating installation.

\section{Characterization}

The concentrations of ions in the sonicated solutions were determined by volumetric titration using Mohrs [14] and complexometric EDTA reagent [15].

The structure of the deposited nanoparticles was characterized by XRD on a Bruker D8 diffractometer (with $\mathrm{Cu} \mathrm{K \alpha}=$ $1.5418 \AA$ radiation). The size of the particles in the working solution was controlled by DLS measurements using a Coulter particle analyzer (Malvern Zetasizer). The morphology of the glasses coated with $\mathrm{NaCl} / \mathrm{CuSO} 4 / \mathrm{KI}$ nanoparticles was studied by SEM using the JEOL-JSN 7000F device. The AFM measurements and imaging were carried out with a Nanoscope V Multimode scanning probe microscope (Digital Instruments, Santa Barbara, CA). All the images were obtained in contact mode with a single NP silicon nitride probe (force constant of $0.58 \mathrm{~N} / \mathrm{m}$, Digital Instruments, Santa Barbara, CA). The scan angle was maintained at $90^{\circ}$ and the images were captured in the retrace direction at a scan rate of $1 \mathrm{~Hz}$. The XPS measurements were performed on a Kratos Axis-HS spectrometer (residual gas pressure of $\sim 5 \times 10^{-10}$ Torr) with monochromatized $\mathrm{Al} \mathrm{K} \alpha$ radiation $(h v=1486.68 \mathrm{eV})$ and a hemispherical analyzer.

\section{Leaching studies}

The leaching studies were performed by placing the coated glasses in $20 \mathrm{~mL}$ of doubly distilled water at room temperature for different periods of time (from 2 to $96 \mathrm{~h}$ ) under continuous stirring. The leaching of salt nanoparticles was estimated by inductively coupled plasma (ICP) analysis on the ULTIMA JY2501 device and examined with AFM.

\section{Results and Discussion}

The current work describes the deposition of $\mathrm{NaCl}, \mathrm{CuSO}_{4}$ and KI nanoparticles on different types of substrates by a one-step, ultrasound-assisted procedure. The results presented here compare the experimental data collected for the three salts. It is important to note that the applied technique is general and the obtained results appeared to be similar to all studied inorganic salts.

\section{Surface properties and morphology}

The first aim of this research was to prove that the nanoparticles produced and deposited onto the polymer and glass substrates have the same composition as the initial salts dissolved in water before the sonication. The structural characterization of the KI nanoparticles deposited on the silicon-wafer slide was performed by XRD, and the result is presented in Figure 2.

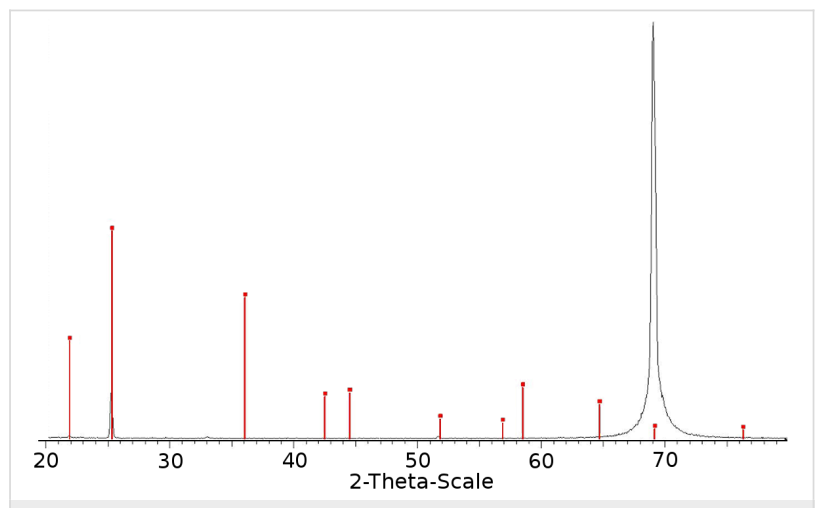

Figure 2: XRD diffraction pattern of NPs of KI coated on a silicon wafer (the red lines indicate literature data (PDF 00-004-0471); the black diffraction lines match well with the KI literature values).

The high-intensity peak at $69.2^{\circ}$ is assigned to reflection lines of the silicon wafer. The peak at $25.106^{\circ}$ corresponds to the (100) reflection line of KI (PDF 00-004-0471). However, not all of the expect peaks for crystalline KI appeared in the spectrum. 
The reason for not observing more than two diffraction peaks of KI may be the small quantity of deposited salt particles on the substrate (less than $4 \%$ ). However, we can also speculate that a peak at $36.1^{\circ}$, which would be the second largest according to PDF 00-004-0471, is missing due to a directional growth of the $\mathrm{KI}$ on the silicon surface.

An additional proof of nanoparticle formation in the sonochemical reaction, based on sonication of the saturated solution of $\mathrm{NaCl}\left(\mathrm{CuSO}_{4}, \mathrm{KI}\right)$, was the formation of sediment that was not immobilized on the glass slide but instead precipitated to the bottom of the reaction vessel and collected at the end of the reaction. The XRD analysis of the collected powder matched well to the diffraction peaks of the crystalline $\mathrm{NaCl}\left(\mathrm{CuSO}_{4}\right.$, $\mathrm{KI}$ ). Figure 3 displays an example of XRD patterns of precipitated $\mathrm{NaCl}$ particles obtained at the end of the sonochemical treatment. The peaks at $2 \theta=27.4,31.8,45.4,53.8,56.4,66.2$, 73.3 and $75.3^{\circ}$ are assigned to the (111), (200), (220), (311), (222), (400), (331) and (420) (PDF 00-005-0628) reflection lines corresponding to the face-centered-cubic structure of $\mathrm{NaCl}$. No peaks characteristic of any impurities were detected. The intensity of the $31.8,45.4,53.8$, and $56.4^{\circ}$ diffraction peaks follow the literature values indicated by the red lines. The diffraction peaks at 66.2 , and $75.3^{\circ}$ deviate from the literature values. This may be a result of (a) another polymorph with (nearly) the same lattice spacings but different electron distribution, or (b), more likely, the possibility that the positional correlation length is different for different lattice directions. In other words, a directed growth of $\mathrm{NaCl}$ crystals emerging as, for

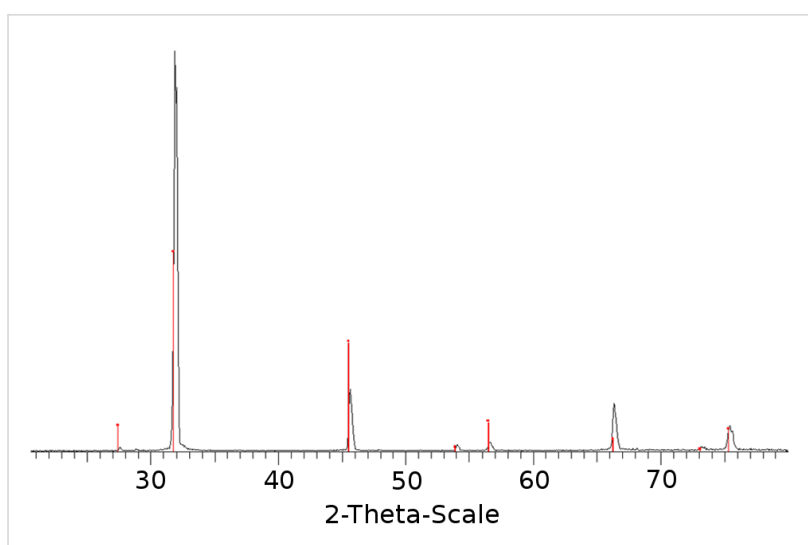

Figure 3: XRD diffraction pattern of $\mathrm{NaCl}$ precipitate collected at the end of sonication (red lines indicate the literature data of PDF 00-0050628, the black diffraction lines were measured for the collected powder).

example, a fibrillar structure may explain these stronger-thanexpected intensities.

The Raman measurements were performed in order to prove the presence of salts on the glass substrates. Figure 4 demonstrates the Raman spectra of the silicon wafer coated with $\mathrm{CuSO}_{4}$. The lines between 200 and $450 \mathrm{~cm}^{-1}$ are assigned to the internal modes of the complex ions $\mathrm{CuSO}_{4}$. The peaks at $475 \mathrm{~cm}^{-1}$, $612 \mathrm{~cm}^{-1}$ and $1107 \mathrm{~cm}^{-1}$ are assigned to the vibration frequencies of $\left(\mathrm{SO}_{4}\right)^{-2}$. In the unit cell of $\mathrm{CuSO}_{4}$, the water molecules occupy three different sites and their geometry is nearly that of the free water molecule $\left(3210 \mathrm{~cm}^{-1}\right)[16]$.

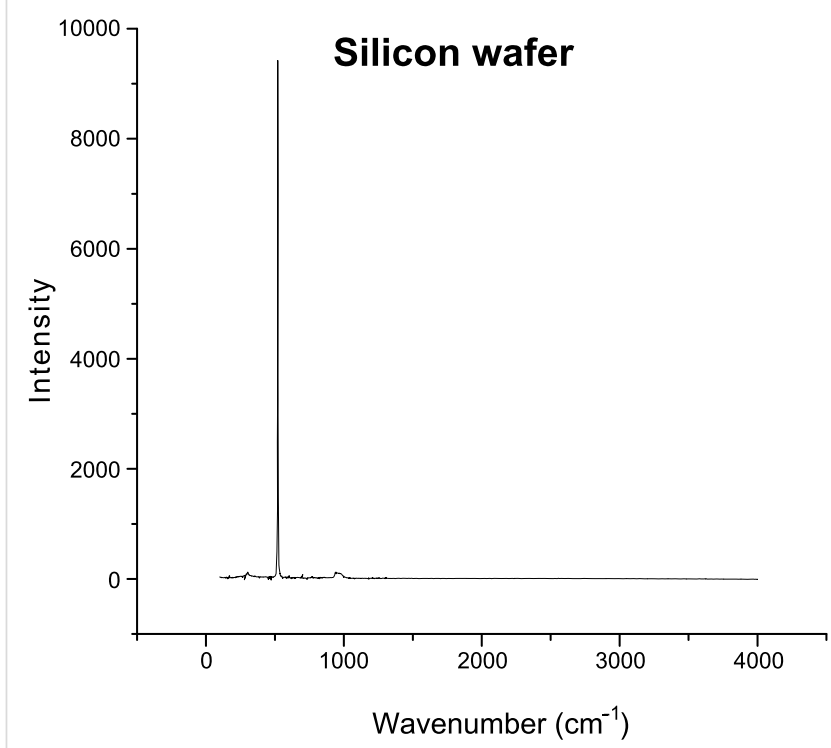

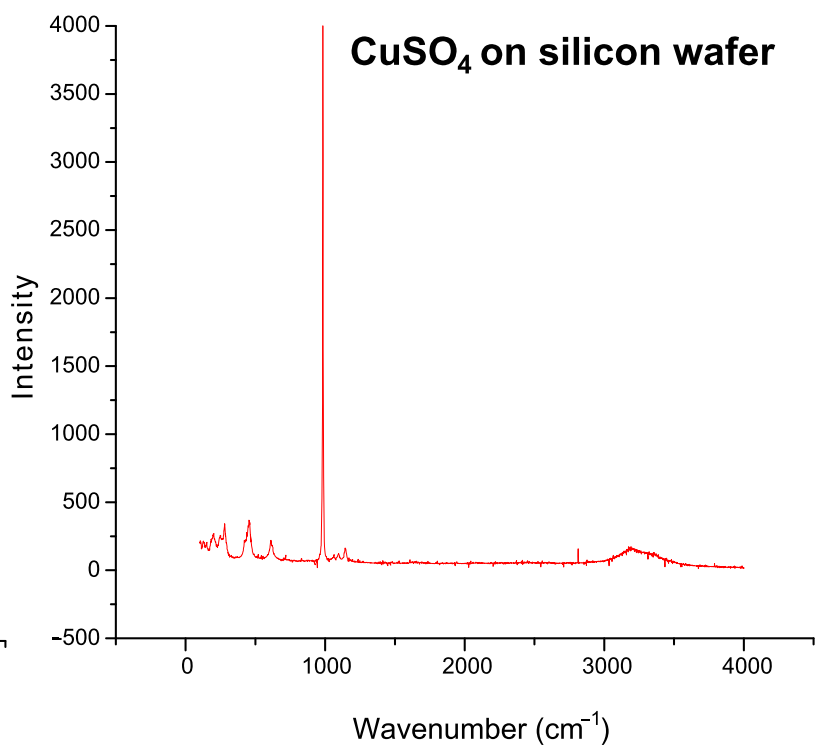

Figure 4: Raman spectrum of a silicon wafer and a silicon wafer coated with $\mathrm{CuSO}_{4}$. 
We also performed the XPS studies of the coated and uncoated glass slides, and the results are presented in Figure 5. There was no use in implementing XPS on the slides coated with $\mathrm{NaCl}$, because the glass itself also contained both $\mathrm{Na}$ and $\mathrm{Cl}$ atoms. Figure 5 demonstrates the XPS spectrum of glass coated with KI nanoparticles. In this case the presence of K and I can only originate from KI, and this result indicated that the salt nanoparticles were attached to the surface of the glass substrate. Similar results were obtained with the samples coated by $\mathrm{CuSO}_{4}$.
To check whether there are differences in the concentrations of ions in the solutions before and after sonication, volumetric titration according to Mohrs method $\left(\mathrm{Cl}^{-}\right)$[14] and complexometric titration with EDTA reagent $\left(\mathrm{Cu}^{2+}\right)$ [15] were performed. The titrations were carried out for the two types of solutions: The solutions that were sonicated without the immersed glass slides and the solutions that were sonicated in the presence of the immersed glass slides. The results obtained for the solutions sonicated without the immersed slides did not
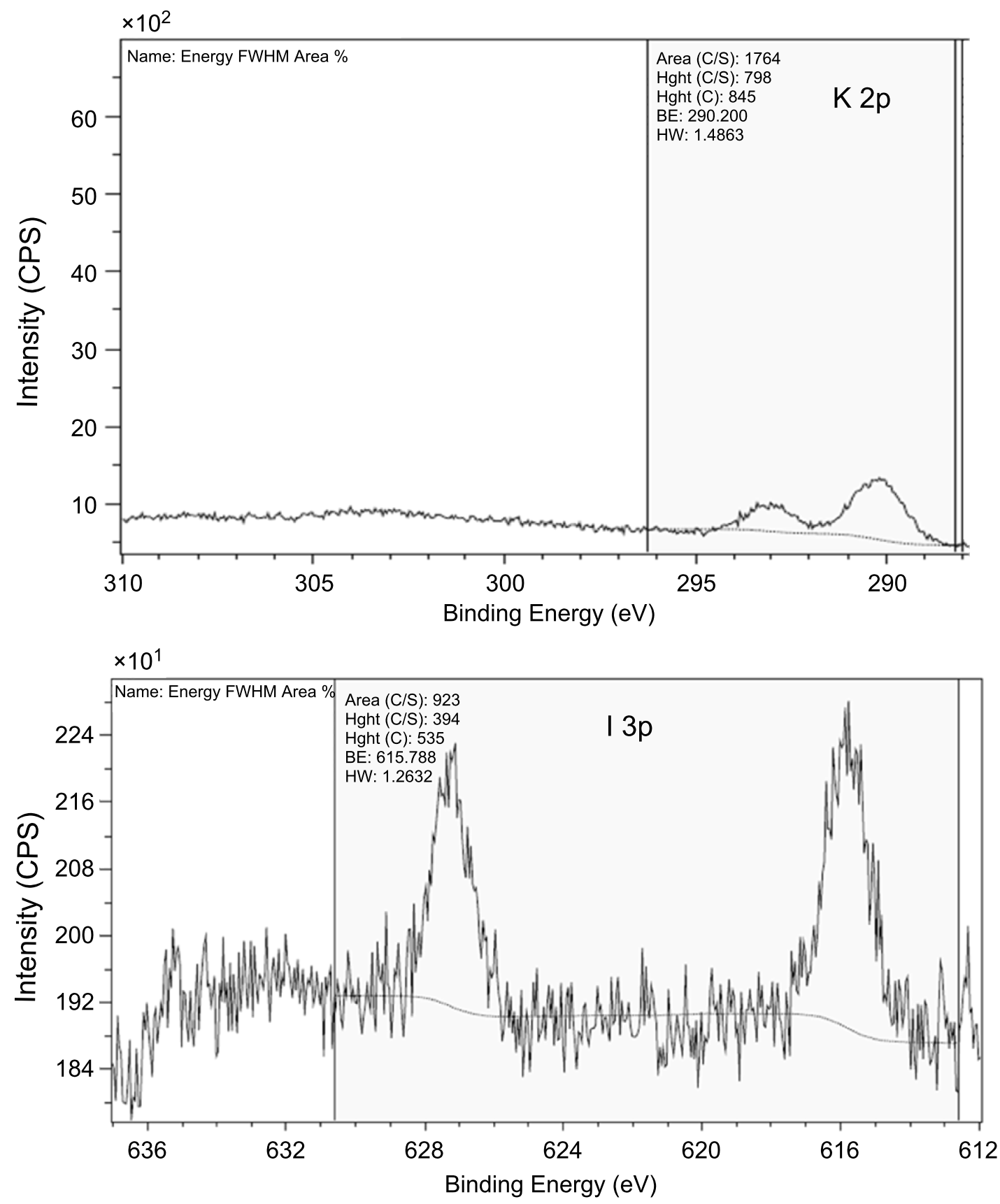

Figure 5: $\mathrm{K}$ (top) and I (bottom) XPS spectra detected from the glass coated with $\mathrm{KI}$. 
show any difference in the concentrations of $\mathrm{Cl}^{-}$or $\mathrm{Cu}^{2+}$ ions compared with their concentrations in the initial solution. At the same time, the titration of the solution sonicated with the immersed slides revealed a decrease in the concentrations of $\mathrm{Cl}^{-}$(for $\mathrm{NaCl}$ ) and $\mathrm{Cu}^{2+}$ ions (for $\mathrm{CuSO}_{4}$ ) in the solutions comparing to their concentrations in the initial solution. This means that part of the solute was retained on the substrate as a deposited layer.

In order to achieve a homogeneous distribution of nanoparticles on the surface, optimization of the sonochemical process was performed by varying the concentration of the reagents and the time for the sonochemical reaction. The influence of the reaction parameters on the obtained results was examined by the SEM method. When the concentration of the starting solution was increased with the other parameters kept unchanged, growth of the deposited particles was observed. For instance, when the concentration of $\mathrm{NaCl}$ in the initial solution was $0.08 \mathrm{M}$ the average size of the deposited nanoparticles was $40 \mathrm{~nm}$ (Figure 6b), whereas when the concentration of the mother solution was increased to $0.3 \mathrm{M}$ larger particles and agglomerates were obtained (Figure 6c).

Another factor that influenced the size of the nanoparticles and the morphology of deposition was the sonication time. Images in Figure $7 \mathrm{~b}$ and Figure $7 \mathrm{c}$ present $\mathrm{CuSO}_{4}$ particles deposited
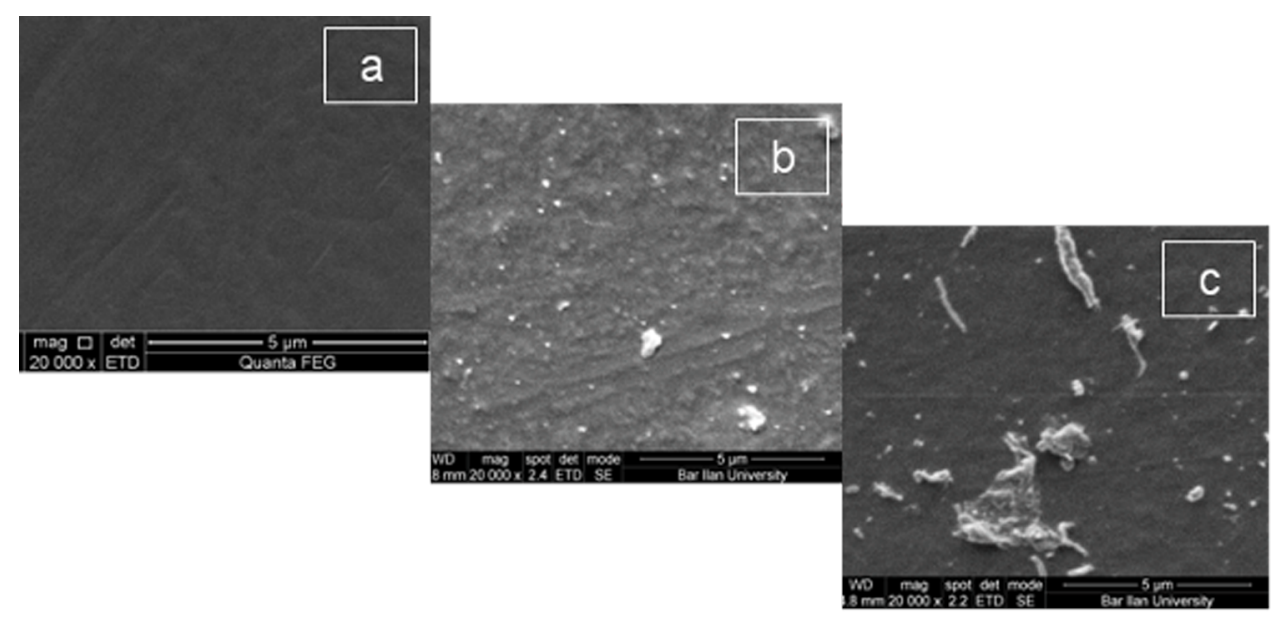

Figure 6: The SEM images of (a) pristine microscope glass slide (magnification 20000x); (b) the glass slide coated with $\mathrm{NaCl}$ nanoparticles after $30 \mathrm{~min}$ reaction in a mother solution of $0.08 \mathrm{M}$ concentration (magnification 20000x); (c) the glass slide coated with $\mathrm{NaCl}$ nanoparticles after 30 min reaction in a mother solution of $0.3 \mathrm{M}$ concentration (magnification 20000x).

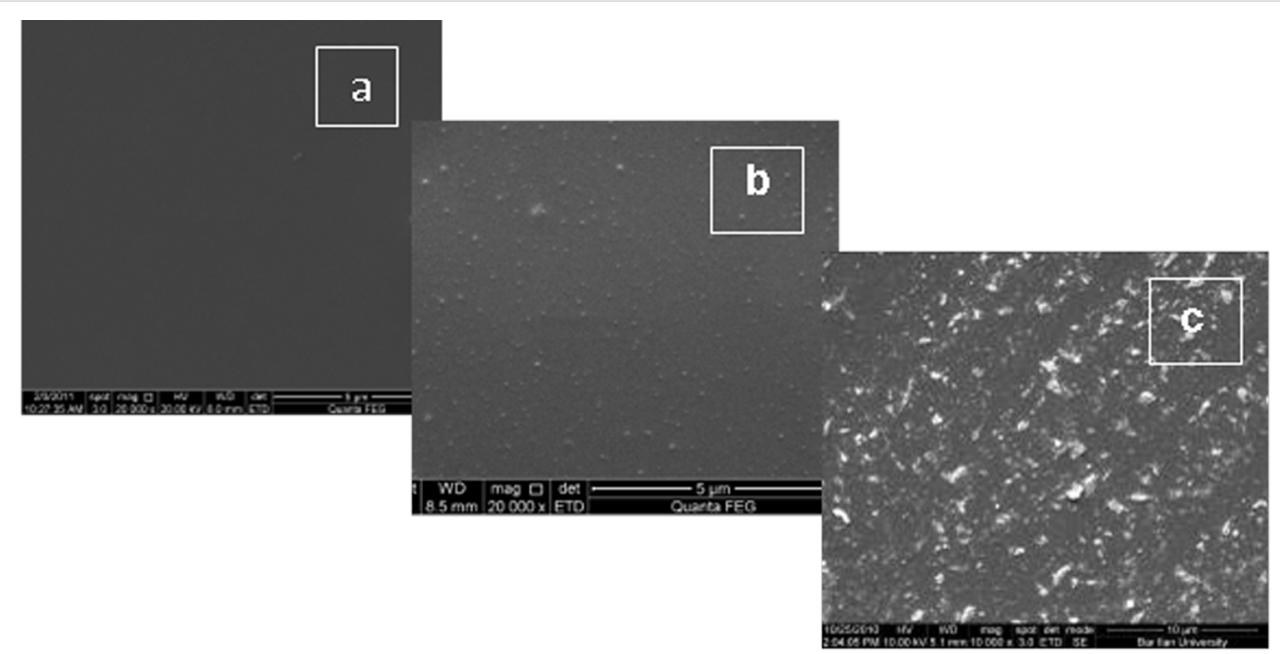

Figure 7: SEM images of (a) parylene-coated glass slide (magnification 20000x); (b) parylene-coated glass slide coated with $\mathrm{CuSO}_{4}$ nanoparticles after $30 \mathrm{~min}$ reaction in a mother solution of $0.125 \mathrm{M}$ concentration (magnification $20000 \times$ ); (c) parylene-coated glass coated with $\mathrm{CuSO}_{4}$ particles after 90 min reaction in a mother solution of $0.125 \mathrm{M}$ concentration (magnification $10000 \times$ ). 
on parylene-coated glass slide from a $0.125 \mathrm{M}$ mother solution, but in the case of Figure $7 \mathrm{~b}$ the exposure time was $30 \mathrm{~min}$ and in Figure 7c 90 min. This obviously shows that an increase in the sonication time leads to enlarged particles and the appearance of aggregates. It was found that the most homogeneous coating could be obtained when the initial concentration of inorganic salts was in the range of $0.05-0.125 \mathrm{M}$ and the ultrasound treatment lasted for $30 \mathrm{~min}$.

This raises the question raised as to whether the nanoparticles were formed in the solution during sonication and then deposited on the surface of the substrate, or whether they crystallized directly on the surface. To confirm the presence of the nanoparticles in the solution and estimate their size, DLS measurements of particles in the solution after the sonication process were performed. The results are summarized in the Figure 8. It was found (data not shown) that the size of the ionic NPs was dependent on the concentration of the ionic material. The use of a higher initial concentration of inorganic salts $(>0.5 \mathrm{M})$ resulted in the appearance of larger particles $(400-500 \mathrm{~nm})$. The DLS of the original was measured and no particles were detected.

The surface morphology of deposed nanoparticles was also characterized by the AFM method. The 3D AFM images of the standard glass slide before and after sonication with $\mathrm{CuSO}_{4}$ are presented in Figure 9. The size of the obtained nanoparticles ranges from $15 \mathrm{~nm}$ to $75 \mathrm{~nm}$. The mean square roughness of the glass with sonochemically deposited nanoparticles is higher $(1.40 \mathrm{~nm})$ than the roughness of the bare microscope glass slide $(0.652 \mathrm{~nm})$. These findings give further proof of the ability of the sonochemical method to deposit inorganic nanoparticles from aqueous solution on the microscope glass slides and parylene-coated glass by a one-step, ultrasound-assisted procedure.

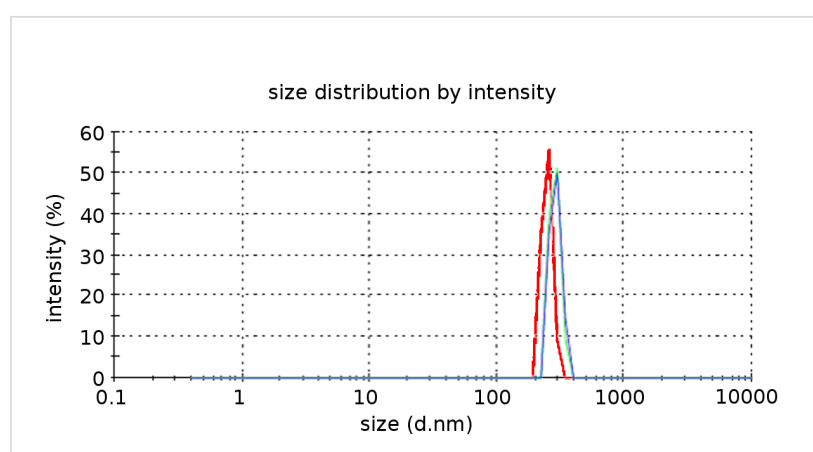

Figure 8: Size distribution measurements of a $0.08 \mathrm{M} \mathrm{NaCl}$ solution after sonication.

A comparison of the SEM and AFM results measured for the same sample, as illustrated in Figure $7 \mathrm{~b}$ and Figure 9b, reveals very similar particles sizes $(70 \mathrm{~nm})$.

\section{Leaching studies}

To examine the strength of anchoring of the NPs to the substrate, some control experiments on the release of the ionic compounds into the surrounding environment were conducted. Namely, we placed the coated glass slides in the doubly distilled water at room temperature for different periods of time (from 2 to $96 \mathrm{~h}$ ) under continuous stirring. The methods we used for revealing the leaching of nanoparticles were ICP and AFM. The ICP measurements showed that after $24 \mathrm{~h}$ the concentrations of $\mathrm{Na}^{+}$and $\mathrm{Cl}^{-}$in water were 0.04 and $0.08 \mathrm{mg} / \mathrm{L}$, respectively, and after $48 \mathrm{~h}$ the concentrations of $\mathrm{Na}^{+}$and $\mathrm{Cl}^{-}$increased to $9.3 \pm 0.4$ and $9.1 \pm 0.3 \mathrm{mg} / \mathrm{L}$, respectively. There is almost no change in the ion concentrations in water between 48 and $96 \mathrm{~h}$. The surface morphologies of the coated glass slides after the leaching experiments, obtained with the AFM method, are shown in Figure 10. The obtained results a

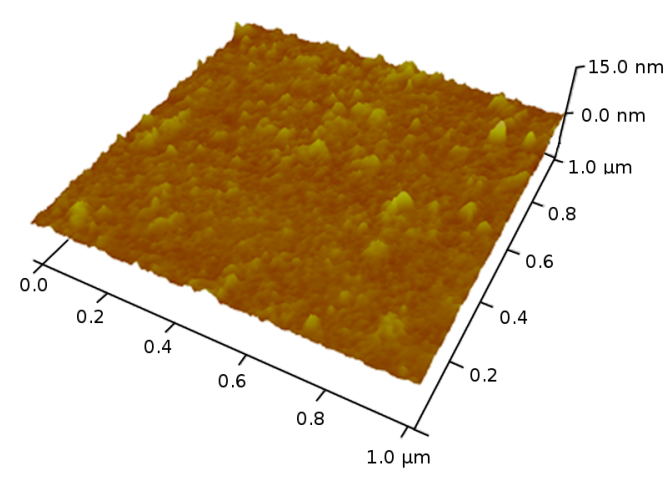

b

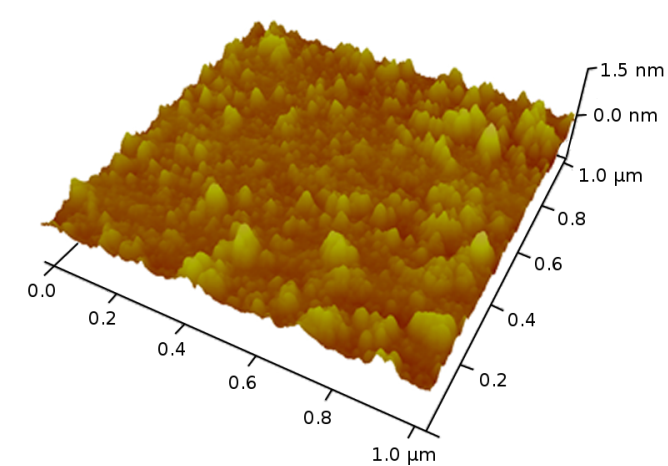

Figure 9: 3D AFM image of the microscope slide after sonication (30 min) in doubly distilled water; (b) 3D AFM image of the microscope slide coated with $\mathrm{CuSO}_{4} \mathrm{NPs}\left(0.125 \mathrm{M} \mathrm{CuSO}_{4}, 30 \mathrm{~min}\right)$. 

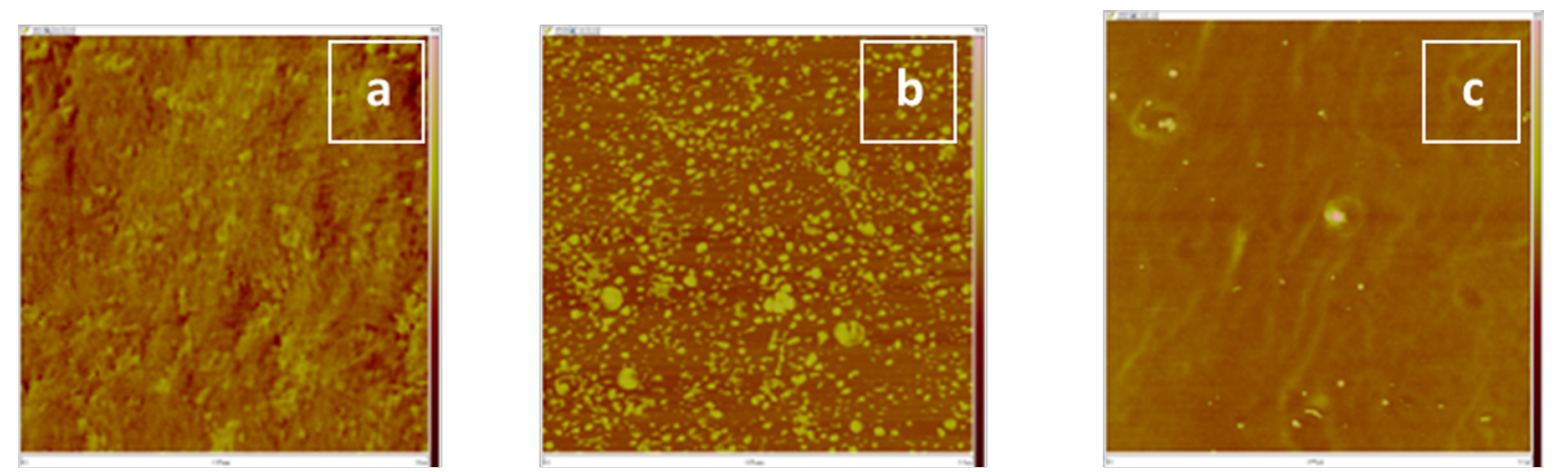

Figure 10: The AFM images of (a) parylene-coated glass slide before the sonication process; (b) parylene-coated glass slide after sonication in the presence of $\mathrm{NaCl}$; (c) the same glass slide after $24 \mathrm{~h}$ of the leaching process.

indicated that after $24 \mathrm{~h}$ of leaching the nanoparticles were still present on the substrates. The complete leaching of nanoparticles occurred after $96 \mathrm{~h}$. The same trend was observed for glass slides coated with $\mathrm{CuSO}_{4}$ and $\mathrm{KI}$ nanoparticles.

\section{Mechanism of the sonochemical deposition}

The sonochemical mechanism by which the nanoparticles were deposited on the substrates was discussed previously and related to the creation of microjets and shock waves as the after effects of acoustic cavitation $[11,12]$.

In the previous studies performed by our group, the deposition of a large variety of nanoparticles on different types of substrates was achieved by using the sonochemical method. In general, there are two main routes for the deposition:

(1) The coating of substrates with nanoparticles that were directly synthesized during the sonochemical reaction (in situ mode of deposition) $[17,18]$.

(2) The coating of substrates with preliminarily synthesized nanoparticles or those purchased from commercial sources ("throwing stones" mode of deposition) [19].

The present work describes an additional route for the deposition of nanoparticles. It was found that the sonication of aqueous solutions of inorganic compounds $\left(\mathrm{NaCl}, \mathrm{CuSO}_{4}, \mathrm{KI}\right)$ resulted in the formation of nanoparticles of the examined salt. In addition, it was found that if this process occurred in the present of glass slide, the nanoparticles formed are deposited onto it.

The proposed mechanism for the described process is the following: When ultrasonic treatment is applied to the aqueous solution of an inorganic salt, the ions of the salt are absorbed on or near the formed acoustic bubbles [20]. When the cavity collapse occurs, the absorbed ions are exposed to extreme, localized conditions of temperature and pressure. As a result, these ions impact one another and nanoparticles of the inorganic salt are produced as a result of the influence of localized higher temperatures and pressures. According to Kordylla et al. [21] the nucleation work needed for crystallization is strongly reduced by the presence of a liquid-gas interface (the bubble surface), therefore the presence of the bubble surface accelerates the crystal nucleation. It is worth mentioning that LepointMullie and co-workers detected visible emission spectra in the vicinity of resonance lines of alkali metals from acoustically cavitating aqueous and 1-octanol solutions of $\mathrm{NaCl}$ and $\mathrm{RbCl}$ [22]. They showed that the emission from the alkali metals arose from the gas phase of the bubbles. The ions reached the hot center of the bubble by the flow of the liquid towards the center upon their collapse. In our case, cavitation occurs not only in pure liquid, but also in liquid near a solid surface (glass slides) and it was found that the presence of a solid surface increases the nucleation rate [23]. The generated high-speed jets of the liquid, formed after the collapse of the bubble, throw the formed nanoparticles at high speed toward the glass slides. The proposed mechanism for the sonochemical deposition of inorganic nanoparticles is presented schematically in Figure 11.

\section{Conclusion}

The $\mathrm{NaCl}, \mathrm{CuSO}_{4}$ and $\mathrm{KI}$ nanoparticles were deposited on the surface of bare or parylene-coated glass slides and silicon wafer or parylene-coated silicon wafer slides by means of ultrasonic (sonochemical) treatment. The size of the observed nanoparticles and their distribution on the substrate were affected by the concentration of the mother solution and the time of exposure to the sonication. The most homogeneous coating was obtained when the initial concentration of inorganic salts was in the range $0.05-0.125 \mathrm{M}$ and the time of the reaction was $30 \mathrm{~min}$. In addition, it was also observed that after $24 \mathrm{~h}$ of leaching the nanoparticles are still present on the slides, and complete 


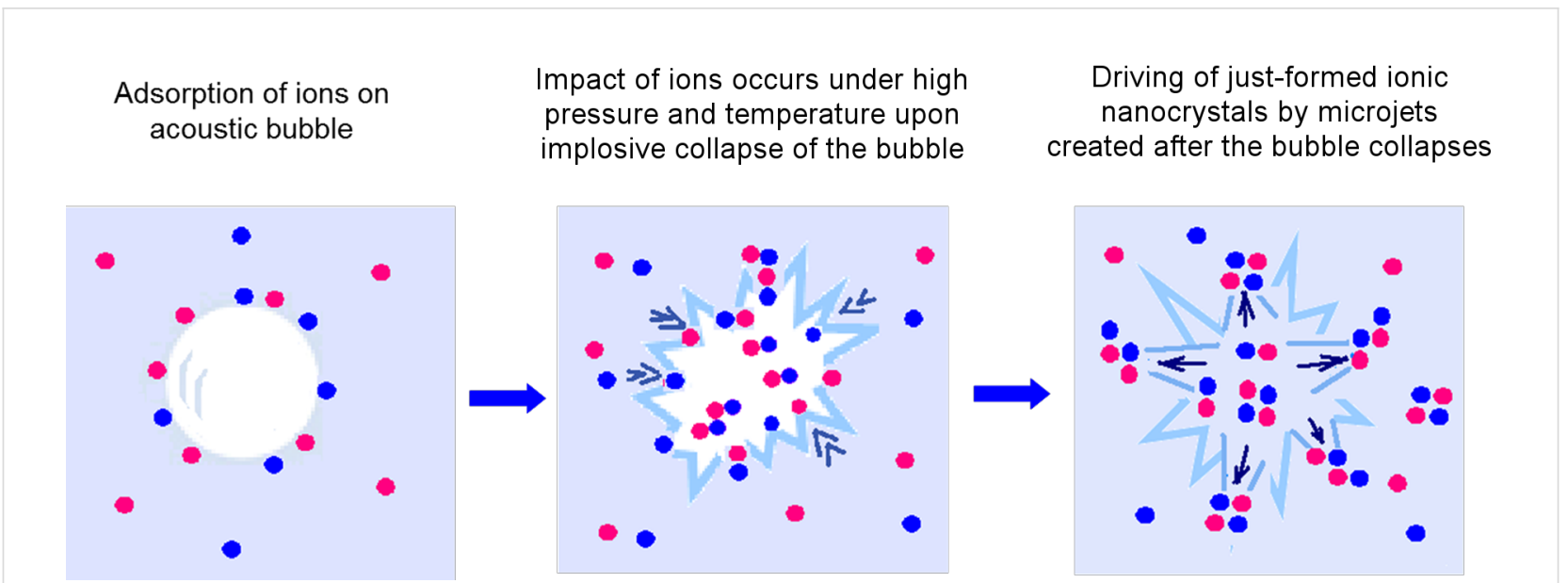

Figure 11: Scheme of the sonochemical deposition of inorganic salt nanoparticles on the solid substrate.

leaching of the nanoparticles occurred only after $96 \mathrm{~h}$. The obtained results clearly demonstrated the efficiency of the ultrasound-assisted method for the deposition of inorganic salt nanoparticles on different types of solid surfaces. This technique can be used to introduce improved properties in terms of electroactivity and conductivity to bare and parylene coated glass slides through such surface modifications.

\section{Acknowledgements}

This research was carried out as part of the activities of the PARYLENES Consortium, Contract No. NMP-2009-1.1-1. PARYLENES, is an IP Project of the $7^{\text {th }}$ EC Program.

\section{References}

1. Peter, S. J.; Miller, M. J.; Yasko, A. W.; Yaszemski, M. J.; Mikos, A. G. J. Biomed. Mater. Res. 1998, 43, 422-427. doi:10.1002/(SICI)1097-4636(199824)43:4<422::AID-JBM9>3.0.CO;21

2. Landis, F. A.; Stephens, J. S.; Cooper, J. A.; Cicerone, M. T.; Lin-Gibson, S. Biomacromolecules 2006, 7, 1751-1757. doi:10.1021/bm0600466

3. Suh, K. Y.; Khademhosseini, A.; Eng, G.; Langer, R. Langmuir 2004, 20, 6080-6084. doi:10.1021/la049217n

4. Wang, Y.; Liu, Z.; Han, B.; Sun, Z.; Zhang, J.; Sun, D. Adv. Funct. Mater. 2005, 15, 655-663. doi:10.1002/adfm.200400201

5. Parfen'eva, L. S.; Smirnov, I. A.; Fokin, A. V.; Misiorek, H.; Mucha, J.; Jezowski, A. Phys. Solid State 2003, 45, 381-385. doi:10.1134/1.1553547

6. Zhang, L.; Cheng, H.-M.; Ma, J.-M. Acta Phys.-Chim. Sin. 2002, 18, 79-81. doi:10.3866/PKU.WHXB20020118

7. Malfatti, L.; Falcaro, P.; Marongiu, D.; Casula, M. F.; Amenitsch, H.; Innocenzi, P. Chem. Mater. 2009, 21, 4846-4850. doi:10.1021/cm9013859

8. Skirtach, A. G.; Kurth, D. G.; Möhwald, H. Appl. Phys. Lett. 2009, 94, 093106. doi:10.1063/1.3093672

9. Lomonosov, A. M.; Meshkov, G. B.; Yaminsky, I. V. http://www.nanoworld.org/russian/SPM2004/SPM2004/187.pdf.
10. Gedanken, A.; Perkas, N. Sonochemical Coatings of Nanoparticles on Flat and Curved Ceramic and Polymeric Surfaces. In Surface Coatings; Rizzo, M.; Bruno, G., Eds.; NOVA Publishers, 2009; pp 213-236.

11. Suslick, K. S.; Choe, S.-B.; Ciclowlas, A. A.; Grinstaff, M. W. Nature 1991, 353, 414-416. doi:10.1038/353414a0

12. Gedanken, A. Ultrason. Sonochem. 2007, 14, 418-430. doi:10.1016/j.ultsonch.2006.08.005

13. Gorham, W. F. J. Polym. Sci., Part A-1: Polym. Chem. 1966, 4, 3027-3039. doi:10.1002/pol.1966.150041209

14. University of Canterbury Home page. http://www.outreach.canterbury.ac.nz/chemistry/documents/chloride_m ohr.pdf (accessed Feb 26, 2012).

15. Husain, A. PHARMACEUTICAL ANALYSIS Theoretical Basis of Analysis: Complexometric Titrations. http://nsdl.niscair.res.in/handle/123456789/771 (accessed Feb 26, 2012).

16. Berger, J. J. Raman Spectrosc. 1976, 5, 103-114. doi:10.1002/jrs.1250050202

17. Perelshtein, I.; Applerot, G.; Perkas, N.; Wehrschetz-Sigl, E.; Hasmann, A.; Guebitz, G. M.; Gedanken, A. ACS Appl. Mater. Interfaces 2009, 1, 361-366. doi:10.1021/am8000743

18. Perkas, N.; Amirian, G.; Girshevitz, O.; Charmet, J.; Laux, E.; Guibert, G.; Keppner, H.; Gedanken, A. Surf. Coat. Technol. 2011, 205, 3190-3197. doi:10.1016/j.surfcoat.2010.11.034

19. Perelshtein, I.; Applerot, G.; Perkas, N.; Grinblat, J.; Hulla, E.; Wehrschuetz-Sigl, E.; Hasmann, A.; Guebitz, G.; Gedanken, A. ACS Appl. Mater. Interfaces 2010, 2, 1999-2004. doi:10.1021/am100291w

20. Sunartio, D.; Yasui, K.; Tuziuti, T.; Kozuka, T.; lida, Y.; Ashokkumar, M.; Grieser, F. ChemPhysChem 2007, 8, 2331-2335. doi:10.1002/cphc.200700426

21. Kordylla, A.; Krawczyk, T.; Tumakaka, F.; Schembecker, G. Chem. Eng. Sci. 2009, 64, 1635-1642. doi:10.1016/j.ces.2008.12.030

22. Lepoint-Mullie, F.; Voglet, N.; Lepoint, T.; Avni, R. Ultrason. Sonochem. 2001, 8, 151-158. doi:10.1016/S1350-4177(00)00030-4

23. Mersmann, A. Crystallization Technology Handbook, 2nd ed.; Marcel Dekker, Inc, 2001; pp 58-67. doi:10.1201/9780203908280 


\section{License and Terms}

This is an Open Access article under the terms of the Creative Commons Attribution License

(http://creativecommons.org/licenses/by/2.0), which permits unrestricted use, distribution, and reproduction in any medium, provided the original work is properly cited.

The license is subject to the Beilstein Journal of Nanotechnology terms and conditions:

(http://www.beilstein-journals.org/bjnano)

The definitive version of this article is the electronic one which can be found at:

doi:10.3762/bjnano.3.30 